\title{
Preface to Vol. 1 Issue No. 2
}

\author{
G. Rigatos $^{1}$ - P. Siano ${ }^{2}$
}

Published online: 7 August 2015

(C) Springer Science+Business Media Singapore 2015

The second issue of the journal comprises articles that provide solutions to nontrivial problems of modelling and control for intelligent industrial systems. In particular, one can distinguish problems associated with intelligent transportation systems (such as automated electric trains, unmanned ground vehicles and unmanned surface vessels), and problems associated with energy management (in buildings and within a smart grid environment). As far as methods for intelligent vehicles are concerned the contribution of the published articles is in assuring that the vehicles will work reliably under changing and uncertain operating conditions. Such vehicles can modify their functional features in a manner that optimally adapts to a varying environment, can cooperate towards the accomplishment of common tasks, can succeed better self-localization and navigation in a dynamic environment, can perform dexterous maneuvers and finally can reach faster and with more precision their destination. As far as methods for energy management are concerned, the contents of the issue show how a coordinated charging scheme for plug-in electric vehicles can reduce stresses in components of the electricity network (such as power transformers) and can mitigate distortion effects and destabilization risks for the smart grid. Moreover, the published articles demonstrate how the monitoring of the functional status of domestic appliances and the optimized management of power consumption in buildings can reduce energy waste

$\triangle$ G. Rigatos

grigat@ieee.org

P. Siano

psiano@unisa.it

1 Unit of Industrial Automation, Industrial Systems Institute, Rion Patras, Greece

2 Department of Industrial Engineering, University of Salerno, Fisciano, Italy as well as the residences' functional costs. The presented results can have also a contribution towards a more balanced functioning of the power grid and towards the prevention of power outages.

In article "Nonlinear H-infinity feedback control for asynchronous motors of electric trains" by G. Rigatos, P. Siano, P. Wira and F. Profumo, a new method for feedback control of asynchronous electrical machines is introduced, with application example the problem of the traction system of electric trains. The control method consists of a repetitive solution of an H-infinity control problem for the train's asynchronous motor, that makes use of a locally linearized model of the motor and takes place at each iteration of the control algorithm. The asynchronous motor's model is locally linearized round its current operating point through the computation of the associated Jacobian matrices. Using the linearized model of the electrical machine an H-infinity feedback control law is computed. The known robustness features of H-infinity control enable to compensate for the errors of the approximative linearization, as well as to eliminate the effects of external perturbations. The proposed control method for the traction system of electric trains is important for improving their performance indexes (e.g. acceleration, maximum speed, motors torque) as well as their safety features.

In article "Distributed control of Unmanned Surface Vessels using the Derivative-free nonlinear Kalman Filter" by G. Rigatos, P, Siano and G. Raffo, a distributed control problem for unmanned surface vessels (USVs) is formulated as follows: there are N USV s wich pursue another vessel (moving target). Each USV can be equipped with various sensors, such as IMU, cameras and non-imaging sensors such as sonar, radar and thermal signature sensors. At each time instant each USV can obtain measurements of the targets cartesian coordinates. Additionally, each USV is aware of the target's distance from a reference monitoring station (coastal 
or satellite monitoring units). The objective is to make the USV s converge in a synchronized manner towards the target, while avoiding collisions between them and avoiding collisions with obstacles in their motion plane. A distributed control law is developed for the USVs which enables not only convergence of the USVs to the goal position, but also makes possible to maintain the cohesion of the multiUSV system. Moreover, distributed filtering is performed, so as to obtain an estimate of the target vessel's state vector. This provides the desirable state vector to be tracked by each one of the USVs. To this end, a new distributed nonlinear filtering method of improved accuracy and computation speed is introduced. This filtering approach, under the name Derivative-free distributed nonlinear Kalman Filter is based on differential flatness theory and on an exact linearization of the target vessels dynamic/kinematic model. The performance of the proposed distributed filtering scheme is compared against the Extended and the Unscented Information filter.

In article "On the use of graph theory for railway power supply systems characterization" by M. Coto, P. Arboleya and C.González-Morán, a DC railway power supply system (RPSS) is presented, in which DC power lines supported by voltage source converters provide electric power for electric trains' traction. This method represents the different elements of the RPSS with a set of subgraphs. After merging these subgraphs, the representative graph of the whole RPSS and its associated adjacency and incidence matrices can be obtained. Once these matrices are computed, Kirchhoffs laws can be easily implemented. The power supply method is applied to a DC light traction system. The AC system that feeds the traction network through power transformers combined with rectifiers is also included. With the proposed approach, the variability problems in the topology and dimensions of the power supply system are overcome. Thus one finally obtains an invariant system, even when the trains change their relative position, or when a new train enters into or exits the system.

In article "Decentralize Coordinated Charging of PlugIn Electric Vehicles in Unbalanced Residential Networks to Control Distribution Transformer Loading, Voltage Profile and Current Unbalance" by M.Moghbel, M. Masoum and A. Fereidouni, utilization of plug-in electric vehicles in residential feeders is examined. The paper aims to alleviate the distortions caused to the smart grid by electric vehicle charging and to assure high power quality standards for the electricity network through the implementation of a coordinated electric vehicles charging algorithm. The approach for improved charging of plug-in electric vehicles is to acquire distribution transformer loading characteristics through smart meters, and to use this information to dynamically coordinate the vehicles' charging times, rates and duration. This method is anticipated to improve the overall system voltage profile, reduce transformers' stress and control current imbalance.

In article "Improvement of traction through $\mathrm{mMPC}$ in linear vehicle dynamics based on electrohydraulic dryclutch", by M. Pisaturo and A. Senatore, a feedback controller is designed based on multiple-model Predictive Controller (mMPC). The controller aims at managing the opening/closing modes in the functioning of a dry-clutch Automated Manual Transmission architecture. This control method enables the vehicle's engine to generate high traction forces. Evaluation tests have been carried out by considering the engine's nonlinear dynamics and disturbances such as time delays. The control scheme was shown to be reliable even when the vehicle performed demanding manoeuvres. Preliminary test results have proven the control method's effectiveness in the case of the electrohydraulic clutch model and have been encouraging about the deployment of the method's application to other transportation systems.

In article, "A Robust Building Energy Management Algorithm Validated in a Smart House Environment" by A. Saha et. al., a core hardware/software platform is developed that enables building operators to address energy consumption needs in an optimised manner, and to manage energy inflow and outflow of the building as part of the smart grid functioning. This paper presents the constituents of a building energy management system that are designed to be robust against communication failures and data errors. Results obtained from the application of the method to a house prototype are reported in this article. The control algorithms which are implemented in the smart house are of the discrete-event type and can be applied for instance to heating, ventilation and air conditioning (HVAC) loads, lighting loads, and electric vehicle chargers. Energy consumption profiles from various seasons of the year were used to validate the efficacy of the proposed energy management approach. The method is primarily addressed to residences, but can be also applied to commercial and industrial buildings.

The selection of articles for this second issue of the Journal of Intelligent Industrial Systems has been the result of a judicious evaluation procedure that was supervised and coordinated by the journal's Editors-in-Chief. The presented articles demonstrate significant achievements in specific topics of industrial systems such as intelligent vehicles and energy management systems. The efficiency and reliability of the considered modelling and control approaches are proven through elaborated mathematical tools and stability analysis methods and are also experimentally confirmed.

The Editors-in-Chief

G. Rigatos and P. Siano 